\section{Reconstruction of a Perineoscrotal Defect Using Bilateral Medial Thigh Fasciocutaneous Flaps}

\author{
Jihoon Yang, Sung Hoon Ko, Suk Joon Oh, \\ Sung Won Jung \\ Department of Plastic and Reconstructive Surgery, Hallym \\ University Sacred Heart Hospital, Hallym University Medical \\ Center, Anyang, Korea
}

\section{Correspondence: Sung Hoon Ko}

Department of Plastic and Reconstructive Surgery, Hallym University Sacred Heart Hospital, Hallym University Medical Center, 22 Gwanpyeong-ro 170beon-gil, Donan-gu, Anyang 431-070, Korea

Tel: +82-31-380-3781, Fax: +82-31-380-5980

E-mail: pshkoh@hallym.or.kr

No potential conflict of interest relevant to this article was reported.

Received: 31 Aug 2012 • Revised: 6 Oct 2012 • Accepted: 7 Oct 2012 pISSN: 2234-6163 • elSSN: 2234-6171

http://dx.doi.org/10.5999/aps.2013.40.1.72 • Arch Plast Surg 2013;40:72-74

Copyright (C) 2013 The Korean Society of Plastic and Reconstructive Surgeon

This is an Open Access article distributed under the terms of the Creative Commons

Attribution Non-Commercial License (http://creativecommons.org/licenses/by-nc/3.0/) which permits unrestricted non-commercial use, distribution, and reproduction in any medium, provided the original work is properly cited.

There are many reasons for skin defects of the perineoscrotal area. These defects can result from severe infection with gangrene and loss of the covering skin. Traumatic avulsions of the scrotal and penile skin are commonly caused by clothing being caught in revolving machinery, automobile versus pedestrian accidents, falls, rare bull-horn avulsion injuries, the excision of scrotal skin diseases and genital burns [1].

Pressure sores of the ischial region have been treated with muscles and skin of the medial part of the thigh. Hirshowitz and Peretz [2] introduced superomedial thigh flaps in the reconstruction of the scrotum and vulva in 1982 . We present two cases using medial thigh fasciocutaneous flaps in the reconstruction of wide skin defects of the perineoscrotal area. The operations were performed under general anesthesia and in the lithotomy position. We first used a Doppler sonogram to mark the external pudendal, superficial femoral, and medial circumflex femoral arteries at the area $5 \mathrm{~cm}$ lateral to the pubic tubercle. Then, a triangular flap was designed. A line was drawn extending from the pubic tubercle to the insertion area of the semitendinosus tendon. The anterior border of the flap may lie a centimeter or so anterior to this line. A triangular flap $9 \mathrm{~cm}$ in width can be safely elevated distally $20 \mathrm{~cm}$ along that border [3].

Dissection begins distally to identify the deep fascia, which must be included with the flap. To preserve the main pedicles, surgeons must not dissect near a line $5 \mathrm{~cm}$ lateral to pubic tubercle. After avoiding the loss of septocutaneous perforators in the dissection, the flap, including the fascia, was raised from the distal aspect, advanced cephalad and rotated medially toward the inguinal canal using the pedicles of the three arteries as a pivot point. Superficial subcutaneous tissues and veins may be included in the pedicle if they do not prevent flap mobilization [4]. Then, the distal end of the flap was rotated toward the anus, and the posterior end of the flap was rotated toward the penis. After 


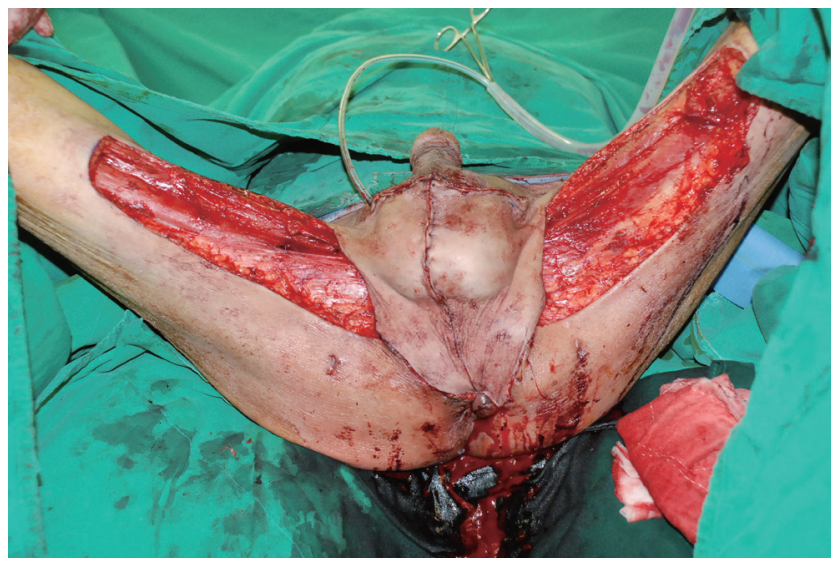

Fig. 2.

Intraoperative photograph of case 1 . The defect was covered with a bilateral medial thigh fasciocutaneous flap.

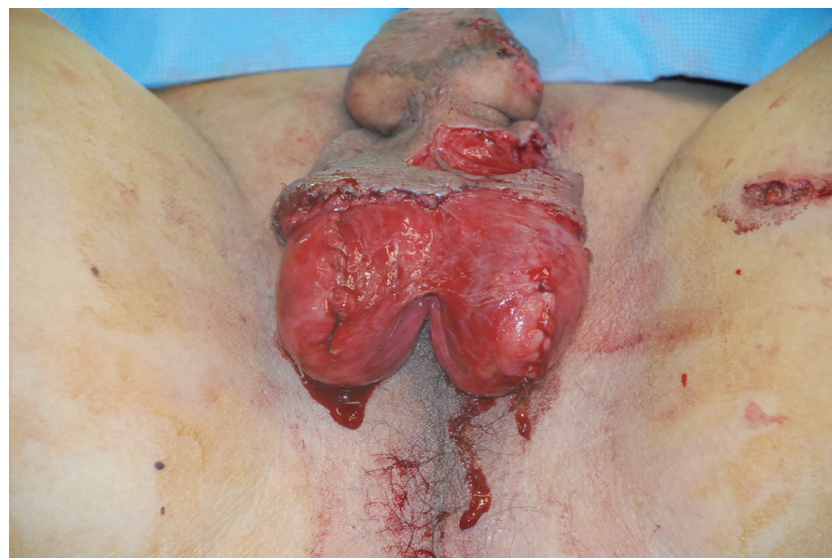

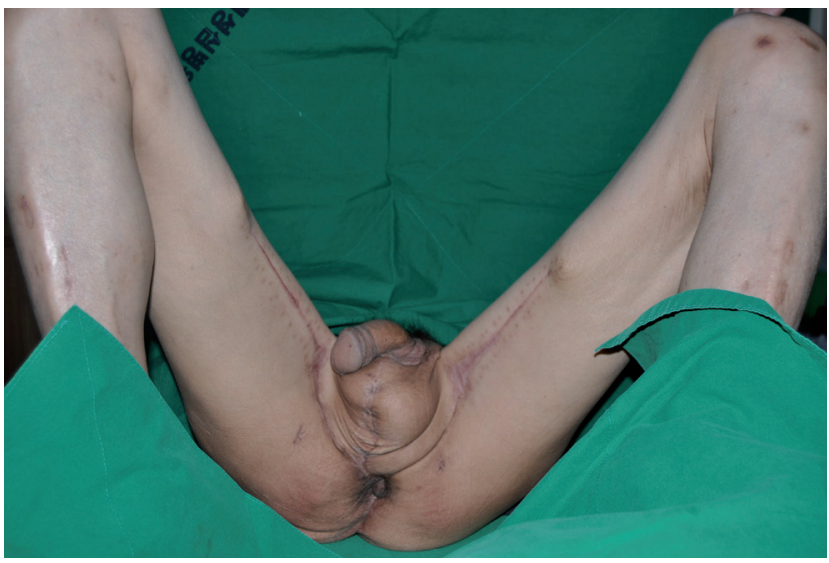

Fig. 3.

Postoperative photograph of case 1 after 6 months. There is no severe scar contracture as can result from a skin graft, and the contour of the reconstructed scrotum looks similar to a normal scrotum.

Fig. 4.

Preoperative photograph of case 2 . The defect size was $10 \times 9 \mathrm{~cm}$. The lower two-thirds of both testes were exposed. coverage of the defect with bilateral flaps, donor site closure could be achieved by primary intention.

A 46-year-old man had a wide perineoscrotal defect due to Fournier's gangrene. He had undergone a colostomy due to anal sphincter dysfunction. The size of the defect was $17 \times 12 \mathrm{~cm}$ and included perineal and scrotal skin (Fig. 1). The defect was covered with a bilateral medial thigh fasciocutaneous flap. The size of each flap was $18 \times 7 \mathrm{~cm}$, and primary closure was performed to close the donor site (Fig. 2). The colostomy failed on postoperative day 2 . The wound became infected due to fecal contamination and dehisced with partial loss of the perianal flap. On postoperative day 20 , the wound was revised under local anesthesia with debridement and primary closure. There was no further flap loss, wound dehiscence, or scar contracture as of 6 months postoperatively (Fig. 3).

A 54-year-old man had a perineal defect due to trauma. He had hepatocellular cell carcinoma, which impaired his coagulation function. The size of the defect was $10 \times 9 \mathrm{~cm}$ (Fig. 4). The defect area was mainly the lower two-thirds of his scrotum and included perineal skin. The defect was covered with bilateral medial thigh fasciocutaneous flaps and remnant scrotum. The size of each flap was $5 \times 6 \mathrm{~cm}$. The wound healed without problems such as hematoma. There were no significant complications or operative site problems as of 3 months postoperatively (Fig. 5). Reconstruction of the perineoscrotal area after complete loss of the overlying skin is a challenging problem for reconstructive surgeons [1]. The fasciocutaneous flap provides durable skin and a large area of soft tissue coverage for reconstructing scrotal and perineal defects. With this thin flap coverage, it produces an acceptable cosmetic effect.

In our cases, the defect areas included both the scrotum and perineum. As mentioned above, surgeons should consider not only the extent of coverage but also aesthetic and functional aspects in cases of scrotal reconstruction.

The medial thigh fasciocutaneous flap is an axial 
Fig. 5 .

Postoperative photograph of case 2 after 3 months. There is no severe scar, as can result from a skin graft, and the contour of the scrotum is exposed naturally due to the thin flaps and remnant scrotal skin.

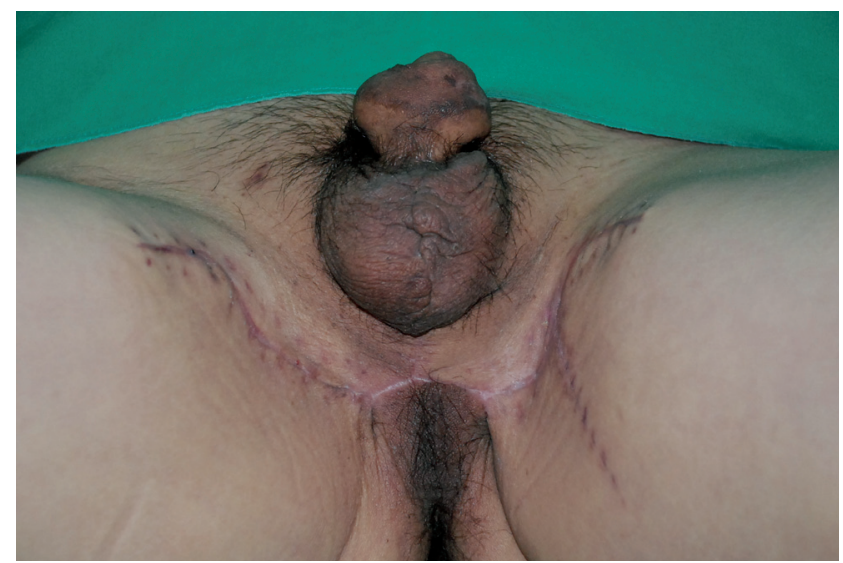

pattern flap that consists of perforators of three arteries: the external pudendal, superficial femoral, and medial circumflex femoral arteries. The presence of axial vessels from the medial femoral circumflex artery and vein has been demonstrated by transillumination at the time of surgery and by cadaveric study $[2,3,5]$. Because of the longitudinal axial interconnections of these three vessels above the deep fascia of the medial thigh, preservation of only the proximal afferents of each pedicle allows for safe elevation of this large, longitudinally-oriented medial thigh fasciocutaneous flap [3]. With this anatomical understanding, the flap can be medially rotated approximately 90 degrees at the lithotomy position, while preserving the axial vessels, to cover the defect. We thought bilateral flaps could maintain the scrotal shape aesthetically better than other unilateral flaps. Compared to other cases of unilateral flap coverage, the midline scar after bilateral flap insertion can substitute for the perineal raphe aesthetically. With thin fasciocutaneous flap coverage, the contour of the reconstructed scrotum looks similar to a normal scrotum.

After flap elevation, the donor site can be closed by primary intention without excessive tension. Aesthetically, the inner part of the thigh is relatively unexposed.

In conclusion, bilateral medial thigh fasciocutaneous flap is a safe and effective procedure in perineoscrotal reconstruction without donor site morbidity for both small and large defects.

\section{References}

1. Ellabban MG, Townsend PL. Single-stage muscle flap reconstruction of major scrotal defects: report of two cases. Br J Plast Surg 2003;56:489-93.

2. Hirshowitz B, Peretz BA. Bilateral superomedial thigh flaps for primary reconstruction of scrotum and vulva. Ann Plast Surg 1982;8:390-6.

3. Hallock GG. Scrotal reconstruction following Fournier's gangrene using the medial thigh fasciocutaneous flap. Ann Plast Surg 1990;24:86-90.

4. Maguina P, Paulius KL, Kale S, et al. Medial thigh fasciocutaneous flaps for reconstruction of the scrotum following Fournier gangrene. Plast Reconstr Surg 2010;125:28e-30e.

5. Cormack GC, Lamberty BG. The blood supply of thigh skin. Plast Reconstr Surg 1985;75:342-54. 